\title{
DIFFERENTIALGEOMETRISCHE METHODEN IN DER PHYSIK
}

\author{
KONRAD BLEULER
}

\section{Einleitung}

Die Gespräche mit Rolf Nevanlinna über mathematische Gesichtspunkte in der heutigen Physik, die sich über sehr viele Jahre hingezogen haben, sind mir in unvergesslicher Erinnerung. Rolf Nevanlinna hatte ein großes Interesse und ein tiefes Verständnis für die entscheidenden mathematischen Gedanken, die der Entwicklung der heutigen theoretischen Physik zugrunde liegen. Er war im Innersten von der Notwendigkeit und der Fruchtbarkeit eines intensiven Austausches zwischen den beiden Gebieten überzeugt. Mit ihm dürfen wir uns fragen: was wäre die heutige theoretische Physik ohne die grundlegenden mathematischen Begriffe wie z. B. der Riemann'sche Raum, Faserbündel, Hilbert'sche Räume, Spektraltheorie, die verschiedenen Gruppen, algebraische Strukturen, Funktionalanalysis und auch Topologie. Diese Begriffe werden in der Physik nicht nur gelegentlich angewendet, sie bilden vielmehr die formale Grundlage der eigentlichen Formulierung der physikalischen Grundgesetze selbst. Man denke dabei besonders an die spezielle und allgemeine Relativitätstheorie, die Formulierung der Quantentheorie mit ihrer neuesten Erweiterung durch die sog. Eichtheorien. In gewissem Sinn wird dabei eine bestimmte mathematische Struktur mit der Deutung einer Klasse physikalischer Tatsachen in wunderbarer Weise in Beziehung gebracht. In diesem Zusammenhang kann man vielleicht die Frage beantworten: Was heißt es, einen bestimmten physikalischen Vorgang theoretisch zu verstehen? Dies bedeutet die Zurückführung einer ganzen, möglichst großen Klasse verwandter physikalischer Erscheinungen auf ein mathematisch wohldefiniertes Gesetz. Der entscheidende Punkt ist hier, daß ein solches Gesetz in gewissem Sinn natürlich erscheint, was nichts anderes heißt, als die Verwendung einer im abstrakten Sinn bereits bekannten mathematischen Struktur. Daß ein solches verstehen oder deuten physikalischer Vorgänge in einem so weiten Rahmen tatsächlich möglich ist, führte Rolf Nevanlinna zur Annahme einer geheimnisvollen Beziehung zwischen der Welt der physikalischen Erscheinungen und der Welt der abstrakten Gedanken. In erstaunlicher Weise können äußerlich ganz verschiedenartige physikalische Erscheinungen tatsächlich auf ein und dieselbe mathematische Struktur zurückgeführt werden. Man 
denke etwa an die Zusammenfassung der elektromagnetischen und der optischen Erscheinungen durch die Maxwell'sche Theorie oder an die Beziehung zwischen Lichtablenkung und Gravitation in der allgemeinen Relativitätstheorie. Dabei ist $\mathrm{zu}$ betonen, daß eine solche übergreifende mathematische Struktur wieder Hinweis und Anlass zur Entdeckung neuartiger physikalischer Tatsachen gibt, sodaß ein wahres Wechselspiel zwischen abstrakten mathematischen Gesichtspunkten und physikalischen Tatsachen entsteht.

Oft erinnerte Rolf Nevanlinna daran, in welch hohem Maße im ersten Viertel dieses Jahrhunderts, insbesondere zur Zeit der Begründung der Relativitätstheorie und der Quantenmechanik, ein Austausch zwischen den beiden Gebieten bestand, indem einige der größten Mathematiker dieser Zeit wie D. Hilbert, F. Klein, J. von Neumann und besonders H. Weyl in entscheidender Weise zur Entwicklung der Physik beigetragen haben. Die beiden großartigen Werke von H. Weyl über Relativitätstheorie und Quantenmechanik gehören auch heute, nach so vielen Jahren, noch zu den bedeutendsten Beiträgen zu diesen Gebieten, aus denen sogar in jüngster Zeit wichtige Anregungen und Gesichtspunkte hervorgingen. Seit dieser großen Zeit haben sich aber die beiden Gebiete zunächst weitgehend unabhängig voneinander entwickelt: Die Physik drang, hauptsächlich aufgrund enorm erweiterter experimenteller Methoden, in ungeahnte Dimensionen, sowohl im Großen wie auch im Mikroskopischen, vor, während die Mathematik ausgedehnte abstrakte Gebiete erschloss. Mit großer Freude erlebte Rolf Nevanlinna, der vor einer Entzweiung der beiden Gebiete in eindringlicher Weise gewarnt hatte, noch einen erneuten Kontakt und Austausch, der gegenwärtig von einigen der bedeutendsten Mathematiker wie M. Atyiah, B. Kostant, R. Bott, S. Sternberg und anderen angeführt wird. Zu den Gebieten, die gegenwärtig vor allem ein gemeinsames Interesse beanspruchen, gehören die sog. geometrische Quantisierung, die konstruktive Feldtheorie, die Supersymmetrie und hauptsächlich die für die Physik grundlegenden Eichtheorien. Diese letzteren stellen im mathematischen Sinn eine außerordentlich natürliche Anwendung differentialgeometrischer Methoden, hauptsächlich in Verbindung mit dem Begriff der Faserbündel, dar. Es soll hier anhand der allereinfachsten Beispiele in Kürze dargelegt werden, wie die Entwicklung bis hin zu den Eichtheorien zustande kam.

\section{Der Aufbau der allgemeinen Relativitätstheorie aus der Elektrodynamik}

Das elektromagnetische Feld erscheint vom experimentellen Standpunkt aus als die Zusammenfassung eines gewöhnlichen polaren Feldes (d. h. das elektrische Feld) und eines axialen (d. h. des magnetischen) Feldes. Die entgegengesetzen Spiegelungscharaktere ergeben sich ohne weiteres aus dem bekannten Ausdruck für die sog. Lorentzkraft, die in das unrelativistische Bewegungsgesetz eines geladenen 
Massenpunktes eingeht:

$$
\vec{K}=e\left(\vec{E}+\frac{1}{c}[\vec{v} \vec{B}]\right)=m \ddot{\vec{x}}
$$

Die Struktur der Maxwell'schen Feldgleichungen ist nun ganz wesentlich durch die sog. Paritätserhaltung oder Invarianz gegenüber Spiegelungen bestimmt:

$$
\operatorname{rot} \vec{B}-\frac{1}{c} \dot{\vec{E}}=\frac{4 \pi}{c} \vec{j} \quad \operatorname{div} \vec{E}=4 \pi \varrho
$$

$$
\operatorname{rot} \vec{E}+\frac{1}{c} \dot{\vec{B}}=0 \quad \operatorname{div} \vec{B}=0
$$

In der Tat gehen in jeder einzelnen Gleichung nur Größen mit demselben Spiegelungscharakter ein und zwar sind unter Annahme der Linearität und der ersten Ordnung (wobei die räumlichen Ableitungen in Form der "hydrodynamischen Begriffe" Rotation und Divergenz auftreten sollen) alle Gleichungen bis auf numerische Faktoren eindeutig bestimmt. Man kann also in diesem Zusammenhang, von einem mathematisch gesehenen, außerordentlich natürlichen Aufbau sprechen.

Von physikalisch weittragender Bedeutung ist aber die Tatsache, daß diese Gleichungen nicht wie diejenigen der klassischen Mechanik gegenüber einer sog. GalileiTransformation

$$
x^{\prime}=x-v t \quad t^{\prime}=t
$$

invariant sind, dafür aber automatisch die grundlegende Lorentz-Transformation in sich bergen:

$$
\begin{gathered}
x^{\prime \mu^{\prime}}=a_{\mu}^{\mu^{\prime}} x^{\mu} \quad a_{\mu}^{\mu^{\prime}} g^{\mu v} a_{v}^{v^{\prime}}=g^{\mu^{\prime} v^{\prime}} \\
\mu=1 \ldots 4 \quad x^{4}=c t \quad g=\left|\begin{array}{lll}
1 & & 0 \\
1 & & \\
& 1 & \\
0 & & -1
\end{array}\right| .
\end{gathered}
$$

Betrachtet man nämlich das Transformationsgesetz eines schiefen Tensors der zweiten Stufe, so sieht man sofort, daß derselbe unter dem Einfluß der speziellen LorentzTransformation, die nur eine räumliche Drehung beschreibt, in der folgenden Weise in einen schiefen 3-dimensionalen Tensor $B_{i k}$ (d. h. einen axialen Vektor) und einen gewöhnlichen Vektor zerfällt:

$$
F_{\mu \nu}=\left|\frac{B_{i k}}{-E_{k}}\right| \frac{E_{i}}{0} \mid \text {. }
$$

Es liegt deshalb nahe, das Maxwell'sche Feld durch einen 4-dimensionalen schiefen Tensor darzustellen, mit dessen Hilfe die Maxwell'schen Gleichungen die folgende 
relativistisch invariante Form annehmen:

$$
\begin{gathered}
\frac{\partial F^{\mu \nu}}{\partial x^{v}}=\frac{4 \pi}{c} J^{\mu} \quad J^{\mu} \rightarrow \vec{j}, c \varrho \\
\left.\frac{\partial F_{\mu \nu}}{\partial x_{\lambda}}\right|_{\text {Ant. }}=0 .
\end{gathered}
$$

Vom Standpunkt der speziellen Relativitätstheorie handelt es sich hier um ein sehr natürliches, aber in keiner Weise ausgezeichnetes System, das nicht durch ähnliche Ausdrücke ersetzt werden könnte. Diese Sachlage ändert sich aber mit einem Schlag, wenn man zur allgemeinen Relativitätstheorie übergeht. Betrachtet man in einer 4-dimensionalen Mannigfaltigkeit ein kovariantes antisymatisches Tensorfeld $F_{\mu \nu}$ zweiter Stufe, sowie eine ebensolche kontravariante Tensordichte $F^{\mu \nu}$, so gilt folgendes: Die beiden Ausdrücke (in (6a) und (6b)) stellen (nach Palais) die einzigen linearen, gegenüber allgemeinen Transformationen invarianten, Operationen dar. Dabei ist die Anti-Symmetrie sogar eine Notwendigkeit und die zweite Operation stellt nichts anderes als die sog. äußere Ableitung dar; die Invarianz des ersten Ausdrucks wird durch den sog. Levi-Civitta'schen $\varepsilon$-Homomorphismus aus dem ersten gewonnen. Damit sind also die beiden zunächst als getrennt aufzufassenden Maxwell'schen Gleichungen in der neuen Form im wesentlichen durch die Annahme der Linearität und allgemeinen Invarianz eindeutig bestimmt. Die Verbindung der beiden Felder geschieht jetzt in natürlicher Weise mit Hilfe eines metrischen Tensors $g$, die ihrerseits eindeutig ist, wenn man verlangt (was physikalisch als natürlich erscheint), daß keine Ableitungen des metrischen Tensors in die Gleichungen eingehen:

$$
F^{\mu v}=\sqrt{-g} g^{\mu \mu^{\prime}} g^{v v^{\prime}} F_{\mu^{\prime} v^{\prime}} .
$$

Man kann in diesem Zusammenhang mit Fug und Recht sagen, daß die Maxwell'schen Gleichungen die Transformationen der allgemeinen Relativitätstheorie in natürlicher Weise in sich bergen.

Es ist interessant von diesem Standpunkt aus auch das Bewegungsgesetz eines geladenen Massenpunktes im elektromagnetischen Feld zu betrachten. Die üblichen mechanischen Bewegungsgleichungen (1) sind nicht invariant gegenüber einer Lorentz-Transformation und sollen deshalb in Übereinstimmung mit (4) in der folgenden Weise geändert werden (hier stehen im Gegensatz zu früher jetzt die Ableitungen $\left({ }^{\circ}\right)$ nach der sog. Eigenzeit, die bis auf einen Faktor die 4-dim. Bogenlänge darstellt):

$$
m \dot{x}^{\mu}=\frac{e}{c} F_{v}^{\mu} \dot{x}^{\nu}
$$

Man kann beweisen, daß diese neuen, sehr viel einfacheren Gleichungen aus der relativistischen Invarianz folgen, unter der Bedingung, daß im Grenzfall kleiner 
Geschwindigkeiten die alten Gleichungen (1) entstehen (d. h. im lokalen Ruhsyst.). Stellt man aber hier jetzt die Frage nach der Invarianz gegenüber den allgemeinen Kooridinatentransformationen, so muß die linke Seite durch den bekannten Zusatzterm der sog. kovarianten Ableitung erweitert werden:

$$
m \check{x}^{\mu}+\Gamma_{\alpha \beta}^{\mu} \dot{x}^{\alpha} \dot{\circ}^{\beta}=\frac{e}{c} F_{v}^{\mu} \stackrel{\circ}{x}^{v}
$$

mit

$$
\Gamma_{\alpha \beta}^{\mu}=g^{\mu \nu}\left\{-\frac{\partial g_{v \beta}}{\partial x^{\alpha}}-\frac{\partial g_{\alpha v}}{\partial x^{\beta}}+\frac{\partial g_{\alpha \beta}}{\partial x^{v}}\right\} .
$$

Dieser vom Standpunkt der Differentialgeometrie notwendige und in gewissem Sinne eindeutig bestimmter Zusatzterm kann physikalisch in natürlicher Weise als Gravitationskraft (die jetzt geschwindigkeitsabhängig ist) interpretiert werden. Dabei ergibt sich zugleich auch die Deutung des metrischen Tensors als verallgemeinertes Gravitationspotential. Stellt man sich in diesem Zusammenhang die Frage, in welcher Weise nun eine invariante Feldgleichung für dieses erweiterte Gravitationspotential angegeben werden könnte, so bietet sich nach David Hilbert das folgende Variationsproblem an:

$$
\delta \int R \sqrt{-g} d v=0 .
$$

Hier bedeutet $R$ den Ausdruck für den Krümmungsskalar, der durch Verjüngung der Krümmungstensore $R_{v \alpha \beta}^{\mu}$ gewonnen wird; dabei gilt für ein beliebiges Vektorfeld $v^{v}$

$$
R_{v \alpha \beta}^{\mu} v^{v}=\left(D_{\left[\alpha^{\prime}\right.} D_{\beta]} v\right)^{\mu}
$$

mit der Abkürzung

$$
\left(D_{\alpha} v\right)^{\mu}=\frac{\partial v^{\mu}}{\partial x^{\alpha}}+\Gamma_{\alpha \beta}^{\mu} v^{\beta} \quad \text { u.s.w., }
$$

d. h. der Krümmungstensor ist durch den Kommutator [...] der kovarianten Ableitung $D$ bestimmt. Unter relativ einfachen Kriterien, die neuerdings sogar verschärft wurden, ist dieser Krümmungsskalar in eindeutiger Weise ausgezeichnet. Dieses Variationsprinzip ergibt die Einstein'schen Feldgleichungen in ihrer definitiven Form, welches automatisch das bekannte Newton'sche Gravitationsgesetz als Grenzfall enthält. In Bezug auf diese in sehr einfacher Form wiedergegebenen Grundgesetze darf man wohl sagen, daß dieselben von einem geeigneten abstrakten Gesichtspunkt als die denkbar natürlichsten erscheinen. Nachdem schon die Maxwell'schen Gleichungen (6) zusammen mit (8) ein riesiges empirisches Material überdecken, ergibt sich jetzt durch eine denkbar natürliche mathematische Erweiterung nach (7), (9) und (11) die richtige Deutung erst in letzter Zeit gut verifizierter Effekte wie Lichtablenkung, Perihelbewegung und Gravitationsabstrahlung. 


\section{Die Gleichungen nach Weyl und Dirac}

Um die Invarianz eines physikalischen Grundgesetzes explizit zum Ausdruck $\mathrm{zu}$ bringen, ist es notwendig, daß sich die verschiedenen physikalischen Größen nach bestimmter Darstellung der unterliegenden Invarianzgruppe transformieren. In der speziellen Relativitätstheorie treten ausschließlich Tensoren verschiedener Strukturen auf, d. h. als Darstellungen kommen im wesentlichen nur die direkten Produkte der ursprünglichen linearen Gruppe vor. Es stellt sich deshalb in natürlicher Weise die Frage, ob es noch wesentlich andere Darstellungen der LorentzGruppe und damit auch der Drehgruppe gibt. Die sog. Spinor-Darstellungen wurden längst vor der Entdeckung der Quantentheorie durch H. Weyl und E. Cartan eingeführt, doch können dieselben wegen ihrem komplexen Charakter erst in der Quantentheorie angewendet werden. Dort spielen dieselben aber eine ganz fundamentale Rolle: Zunächst denke man an die 2-dimensionale Darstellung $S$ (dieselbe ist aus bekannten Gründen nicht eindeutig) der gewöhnlichen Drehgruppe. Dieselbe stellt den Transformationscharakter des 2-komponentigen Schrödinger'schen Wellenfeldes, d. h. der Pauli'schen Theorie, dar und spielt damit eine fundamentale Rolle in der unrelativistischen wellenmechanischen Bearbeitung aller Partikel mit Spin 1/2. Als charakteristischer Term, der die Kopplung des Spins mit einem äußeren Magnetfeld beschreibt, steht

$$
H^{\prime}=(\vec{B} \vec{\sigma})
$$

wobei $\vec{\sigma}$ die drei Pauli'schen Matrizen $\sigma_{k}(k=1 \ldots 3)$ darstellt, die einerseits die Darstellungen der infinitesimalen Drehungen $\delta \varepsilon_{k l}$ erzeugen

$$
\delta S=\frac{1}{2} \delta \varepsilon_{k l} \sigma_{k} \sigma_{l}
$$

und andererseits die fundamentale Beziehung bezüglich einer 3-dimensionalen Drehung $a_{k l}$ erfüllen

$$
S^{-1} \sigma_{k} S=\sum_{l} a_{k l} \sigma_{l}
$$

die in natürlicher Weise zur Invarianz des oben angegebenen Störungsterms führt. Hermann Weyl hat nun in seinem grundlegenden Buch über Quantenmechanik und Gruppentheorie die Erweiterung dieser Darstellung für die Lorentz-Gruppe verwendet. Zu diesem Zweck wird (1) durch Hinzufügen folgender Terme erweitert:

$$
\delta S=\frac{1}{2} \delta \varepsilon_{4 k} \sigma_{4} \sigma_{k}
$$

mit

Zugleich erweitert sich dann (2) zu

$$
\sigma_{4}=I
$$

$$
S^{+} \sigma^{\mu} S=a_{v}^{\mu} \sigma^{\nu}
$$


( $S$ ist wegen (3) nicht mehr unitär; $S^{+}$bedeutet den hermitisch konjugierten Operator).

Diese Beziehung führt in natürlicher Weise zu der denkbar einfachsten relativistisch-invarianten Feldgleichung für ein zwei-komponentiges Feld $\varphi$

$$
D \varphi \equiv\left(\frac{\partial}{\partial x^{\mu}} \sigma^{\mu}\right) \varphi=0
$$

d. h. die sog. Weyl'sche Gleichung. Diese Gleichung ist wohl invariant gegenüber der eigentlichen Lorentz-Gruppe, nicht aber gegenüber Spiegelungen. Dies geht ohne weiteres aus der Tatsache hervor, daß einerseits im unrelativistischen Fall die räumliche Spiegelung durch den Einheitsoperator festgelegt ist und andererseits die eigentlichen Lorentztransformationen mit den räumlichen Spiegelungen nicht vertauschen. Aus diesem Grunde schien diese Gleichung in der Physik keine Anwendung zu haben, obwohl Hermann Weyl - im Gegensatz zu der damaligen Ansicht von Wolfgang Pauli - betonte, daß eine Spiegelungs-Invarianz keine logische Notwendigkeit darstellt. Diese Lage änderte sich mit einem Schlag, als mehr als 20 Jahre später bei der Vermessung sog. Beta-Prozesse eine Verletzung des Spiegelungs-Invarianz experimentell tatsächlich festgestellt wurde. In diesem Zusammenhang ergab sich in ganz natürlicher Weise die Verwendung der Weyl'schen Gleichung als Wellengleichung der Neutrinos, die in den genannten Prozessen eine maßgebende Rolle spielen (Die Weyl'sche Gleichung erzwingt zugleich - wegen der Nichtunitarität von $S$ - die experimentell bekannte Tatsache, daß die Neutrinos die Masse Null besitzen). Aus all diesen Gründen darf man wohl sagen, daß die Weyl'sche Gleichung, die zugleich die Grundlage der wesentlich erweiterten modernen Theorien über schwache Wechselwirkung in Verbindung mit der Elektrodynamik bildet, ein eindrückliches Beispiel dafür ist, daß mathematisch natürlich erscheinende Strukturen in der Physik von grundlegender Bedeutung sein können.

Auf der anderen Seite bildet aber die Weyl'sche Gleichung zugleich den elementaren Baustein zum Aufbau der berühmten Dirac'schen Gleichung, in der einerseits die Paritätserhaltung garantiert ist und zugleich ein Masse-Term auftritt. (Dies ist notwendig zur wellenmechanischen Beschreibung einer großen Klasse bekannter Partikel.) $\mathrm{Zu}$ diesem $\mathrm{Zweck}$ beachtet man, daß es eine zweite (in gewissem Sinn gespiegelte) Erweiterung der Drehgruppe gibt. Sie wird erhalten, indem man das Vorzeichen von $\sigma_{4}$ ändert wodurch man eine zweite Darstellung $\tilde{S}$ der Lorentzgruppe erhält, die mit der ursprünglichen in der folgenden Weise zusammenhängt:

$$
\tilde{S}=\left(S^{+}\right)^{-1} \text {. }
$$

Damit wird eine zweite Weyl'sche Gleichung für das neue Feld $\tilde{\varphi}$ konstruiert; mit $\tilde{\sigma}^{4}=-\sigma^{4}, \tilde{\sigma}^{k}=\sigma^{k}$ heißt dieselbe (sog. Antineutrinogleichung)

$$
\tilde{D} \tilde{\varphi} \equiv\left(\frac{\partial}{\partial x^{\mu}} \tilde{\sigma}^{\mu}\right) \tilde{\varphi}(x)=0 .
$$


In der bekannten Weise können nun die beiden verschiedenen Weyl'schen Spinoren in ein 4-komponentiges System zusammengefasst werden, das nun vermöge der ober angegebenen Beziehung nicht nur gegenüber eigentlichen Lorentz-Transformationen (vermöge (6)) invariant ist, sondern bei Spiegelung durch den Austausch der beiden Spinoren ebenfalls in sich übergeht:

$$
\begin{aligned}
& D \varphi+i m \tilde{\varphi}=0 \\
& \tilde{D} \tilde{\varphi}+i m \varphi=0 .
\end{aligned}
$$

Daraus entsteht durch die Einführung einer 4-kompon. Größe $\psi(\varphi, \tilde{\varphi} \rightarrow \psi)$ die Dirac'sche Gleichung in der bekannten Form

$$
\left(\frac{\partial}{\partial x^{\mu}} \gamma^{\mu}\right) \psi+m \psi=0 .
$$

Es liegt also in gewissem Sinne bei diesem Aufbau der Dirac'schen Gleichung ein ähnliches Konstruktionsprinzip wie in den Maxwell'schen Gleichungen zugrunde, indem Größen mit verschiedenem Transformationscharakter in geeigneter Weise kombiniert werden, wobei gleichzeitig die Möglichkeit entsteht, einen freien Parameter $m$ in die Gleichungen einzuführen, der physikalisch die Bedeutung der Masse hat. Es ist in diesem Zusammenhang interessant festzustellen, daß in allen physikalischen Prozessen, in denen Partikel (vom Spin 1/2) mit nicht-verschwindender Masse beteiligt sind, die Parität immer erhalten bleibt.

\section{Die Eichtheorien}

Die Dirac'sche Gleichung wird für den Fall der Wechselwirkung mit dem elektromagnetischen Feld in der folgenden Weise erweitert:

$$
\gamma^{\mu}\left(\frac{\partial}{\partial x^{\mu}}+i g A_{\mu}\right) \psi+m \psi=0
$$

d. h., der Operator des Impulses wird mit Hilfe des Vektor-Potentials $A$ des elektromagnetischen Feldes ergänzt (dasselbe existiert lokal aufgrund II, (6b)).*) Einerseits wird dadurch in natürlicher Weise die allgemeine Lorentz-Invarianz erhalten und andererseits die Beziehung zur klassisch-relativistischen Bewegungsgleichung II (8) im sog. klassischen Grenzfall sichergestellt. Es ist aber von entscheidender Bedeutung, daß die so gewonnene Gleichung noch eine zusätzliche, sehr allgemeine Invarianzforderung, nämlich die sog. Eich-Invarianz, erfüllt. Man stellt fest, daß die

*) $g$ steht für die Ladung $e$. 
physikalische Deutung der Dirac'schen Wellenfunktion für die Bildung einer neuen Wellenfunktion durch hinzufügen eines raum-zeit-abhängigen Phasenfaktors nicht geändert wird:

$$
\psi \rightarrow \psi^{\prime} e^{i g \Lambda(x)} .
$$

Man muß deshalb verlangen, daß diese neue Wellenfunktion im wesentlichen dieselbe Wellengleichung erfüllen soll. Durch Einsetzungen ergibt sich aber sogleich, daß die neue Wellengleichung lautet:

$$
\gamma^{\mu}\left(\frac{\partial}{\partial x^{\mu}}+i g\left(A_{\mu}+\frac{\partial \Lambda}{\partial x^{\mu}}\right)\right) \psi^{\prime}+m \psi^{\prime}=0
$$

d. h., das Vektorpotential ist durch hinzufügen eines Gradienten verändert worden. Diese Änderung ist aber aufgrund der physikalischen Deutung des Potentials

$$
F_{\mu v}=\frac{\partial A_{v}}{\partial x^{\mu}}-\frac{\partial A_{\mu}}{\partial x^{v}}
$$

nicht nur erlaubt, sondern auch ganz natürlich. Man sieht damit, daß das angegebene Kopplungsschema, das bereits durch die klassische Physik in gewissem Sinn festgelegt wurde, auch aufgrund der Eich-Invarianz weitgehend bestimmt ist. Von mathematischer Seite kann diese Tatsache in der folgenden Weise aufgefasst werden: Man betrachte den Operator

$$
D_{\mu}=\frac{\partial}{\partial x^{\mu}}+i g A_{\mu}
$$

als eine lineare Übertragung in dem einfachen Faserbündel, das durch die LorentzMannigfaltigkeit und die komplexen Zahlen als Faser gegeben ist. Die zu dieser Übertragung gehörige Krümmungsform $R$

$$
R_{\mu v}=\left[D_{\mu}, D_{v}\right]
$$

ergibt dann gerade das (eich-invariante) elektromagnetische Feld (4), in formaler Analogie zu II (12).

Dieses Schema wird nun zur Aufstellung der heutzutage grundlegenden Eichtheorien in natürlicher Weise erweitert. Dies soll abschließend am Beispiel der sog. Yang-Mills-Theorie angegeben werden. Als Faser wird in diesem Fall die 2-dimensionale komplexe Ebene angenommen und als Eichgruppe im Gegensatz $\mathrm{zu}$ der oben (nach (2)) verwendeten SU (1) wird jetzt SU (2) verwendet. Unter Einführung der Pauli'schen Matrizen, die in diesem Fall die Bedeutung des sog. Isotopen-Spins haben und mit $\vec{\tau}$ bezeichnet werden, lauten diese Gleichungen zunächst aus physikalischen Gründen :

$$
\gamma^{\mu}\left(\frac{\partial}{\partial x^{\mu}}+i g\left(\vec{\tau} \vec{A}_{\mu}\right)\right) \psi+m \psi=0
$$


wobei jetzt die $A_{\mu} 3$ Komponenten besitzten die als Iso-Vektor $\vec{A}_{\mu}$ zusammengefasst werden und $\psi$ jetzt als Element der neuen Faser eine komplexwertige 2-komponentige Größe ist, auf welche die Matrizen $\tau$ in natürlicher Weise wirken. Der neuartige Wechselwirkungsterm ist mit ganz anderer physikalischer Bedeutung ana$\log$ zu III (o) gebaut. Er bildet u. a. die formale Grundlage zu der erfolgreichen Salam-Weinberg'schen Theorie, in welcher schwache und elektromagnetische Wechselwirkungen zusammengefasst werden. Eine infinitesimale Eich-Transformation hat jetzt die Form:

$$
\psi=\psi^{\prime} e^{i g(\tilde{\tau} \tilde{\omega})}
$$

wobei $\vec{\omega} \equiv \vec{\omega}(x)$ die raum-zeit-abhängige infinitesimale Drehung im Isospin-Raum bedeutet. Die Invarianz aller aus der Theorie abzuleitenden physikalischen Größen gegenüber dieser sog. lokalen Eich-Transformation (7) wird nun als grundlegendes Prinzip aufgestellt. Aufgrund der bekannten Vertauschungsrelationen der $\tau$-Matrizen erhält man jetzt für das eich-transformierte $\psi$ Feld die Gleichung

$$
\gamma^{\mu}\left(\frac{\partial}{\partial x^{\mu}}+i g\left(\vec{\tau}\left(\vec{A}_{\mu}+\frac{\partial \vec{\omega}}{\partial x^{\mu}}+\left[\vec{A}_{\mu} \vec{\omega}\right]\right)\right)\right) \psi^{\prime}+m \psi^{\prime}=0
$$

Um auch hier wieder zu einer ,eich-invarianten“ Feldstärke $F$ zu kommen, bildet man wie oben die zu der erweiterten Übertragung

$$
D_{\mu} \equiv \frac{\partial}{\partial x^{\mu}}+i g\left(\vec{\tau} \vec{A}_{\mu}\right)
$$

gehörigen Krümmungsform

$$
\left(\vec{F}_{\mu \nu} \vec{\tau}\right)=\left[D_{\mu}, D_{v}\right]
$$

mit

$$
\vec{F}_{\mu \nu}=\frac{\partial \vec{A}_{v}}{\partial x^{\mu}}-\frac{\partial \vec{A}_{\mu}}{\partial x^{v}}+\left[\vec{A}_{\mu}, \vec{A}_{v}\right]
$$

Dieser Ausdruck enthält gegenüber (4) einen charakteristischen nicht-linearen Zusatzterm, der vom nicht-abel'schen Charakter der erweiterten Eichtransformation (7) herrührt. Er ist notwendig, um den auch direkt verifizierbaren kovarianten Charakter von $\vec{F}$ gegenüber Eichtransformationen zu garantieren. In Analogie zum bekannten elektromagnetischen Fall kann man nun aus dieser „eich-kovarianten“ Feldstärke einen eich-invarianten Skalar $L$ bilden

$$
L=\left(\vec{F}_{\mu \nu} \vec{F}^{\mu v}\right)
$$

der als Lagrange-Funktion für das ursprüngliche Feld $\vec{A}_{\mu}$ verwendet wird. Wegen dem nicht-linearen Zusatzterm (in (11)) enthält diese Lagrange-Funktion im Gegen- 
satz zur Elektrodynamik einen Term vierter Ordnung in $\vec{A}$, sodaß die aus der Lagrange-Funktion abzuleitende Feldgleichung einen nicht-linearen Charakter erhält. Auf der einen Seite sind diese Feldgleichungen (schon im freien Fall) von größtem mathematischem Interesse, da es möglich war, die Lösungsgesamtheit (im sog. euklidischen Fall, d. h. $g_{\mu \nu}=I$ ) vollständig zu übersehen. Eine Erweiterung dieser Eich-Theorien auf die Gruppe SU (3) stellt gleichzeitig die mathematische Grundlage der sog. starken Wechselwirkung, d. h. des Systems aus Quarks und Gluonen, dar. Damit wären in sehr vereinfachter Form im wesentlichen alle mathematischen Grundstrukturen der heutigen Physik zusammengefasst; es sollte dabei nochmals betont werden, daß dieselben in allen Fällen in ihrer mathematischen Form außerordentlich natürlich erscheinen.

Universität Bonn

Nussallee 14-16

D—5300 Bonn

West-Deutschland

Eingegangen am 6. März 1981 